\title{
Nivel óptimo de proteína dietaria para juveniles de lenguado Paralichthys adspersus (Pisces: Pleuronectiformes)
}

\author{
Optimal dietary protein level for flounder Paralichthys adspersus juveniles \\ (Pisces: Pleuronectiformes)
}

\author{
Nicole Piaget ${ }^{1}$, Pedro Toledo ${ }^{2,3}$, Alfonso Silva ${ }^{2}$ y Alonso Vega ${ }^{1,3,4}$

\begin{abstract}
${ }^{1}$ Departamento de Biología Marina, Facultad de Ciencias del Mar, Universidad Católica del Norte, Casilla 117, Coquimbo, Chile. piaget@ucn.cl

${ }^{2}$ Departamento de Acuicultura, Facultad de Ciencias del Mar, Universidad Católica del Norte, Casilla 117, Coquimbo, Chile ${ }^{3}$ CEAZA, Centro de Estudios Avanzados de Zonas Áridas, Coquimbo, Chile

${ }^{4}$ Programa de Doctorado en Biología y Ecología Aplicada, Universidad Católica del Norte, Facultad de Ciencias del Mar, Coquimbo, Chile
\end{abstract}

\begin{abstract}
This study establishes the dietary protein level for maximum growth in Paralichthys adspersus juveniles. According with this purpose, three diets based on fish meal were formulated with increasing protein levels ( 48,57 and $60 \%$ ), which were compared with a commercial diet (54\%protein; BIOMAR S. A.). Furthermore, the optimal protein level were determined and compared through bioassays using growth indicators, fitness, and regression analysis. A total of 156 juveniles ( $131.3 \pm 23 \mathrm{~g}$ ), separated in 13 individuals per group, were maintained in black conical tanks with a flat base, with $5.5 \mathrm{~kg} \mathrm{~m}^{-2}$ initial densities, and with a circulation system of open water for 175 days. The fish were fed daily to satiety twice per day with diets based on fish meal and oil, starch, vitamins and minerals. Based on the results of this study, we conclude that the optimal dietary protein level was obtained in diets with 54 and $57 \%$ of protein. If production costs are prioritized in flounder culture, the $54 \%$ protein diet is optimal for juveniles. However, considering the flounder growth, then $57 \%$ protein diet contains the optimal level. This study is a precedent for further research on the nutritional requirements of $\mathrm{P}$. adspersus in order to improve their growth in captivity.
\end{abstract}

Key words: Aquaculture, nutrition, fish, food, indicators

Resumen. - Este estudio establece el nivel óptimo de proteína dietaria para obtener el máximo crecimiento de juveniles de Paralichthys adspersus. Con este propósito, se formularon 3 dietas con niveles crecientes de proteína en base a harina de pescado (48, 57 y 60\%), las cuales fueron comparadas junto a una dieta comercial (54\%de proteína; BIOMAR S. A. ). El nivel óptimo de proteína fue determinado a través de bioensayos utilizando indicadores de condición, utilización del alimento y análisis de regresión. Un total de 156 juveniles $(131,3 \pm 23 \mathrm{~g})$, divididos en grupos de 13 individuos, fueron mantenidos en estanques cónicos negros de base plana con densidad inicial de $5,5 \mathrm{~kg} \mathrm{~m}^{-2}$, y con un sistema de circulación de agua abierto por un periodo de 175 días. Los peces fueron alimentados diariamente dos veces al día a saciedad. En base a los resultados de este estudio se concluye que el nivel óptimo de proteína dietaria fue obtenido con dietas de $54 \%$ y $57 \%$ de proteína. Si los costos productivos son priorizados en el cultivo del lenguado, entonces la dieta con $54 \%$ de proteína es óptima para alimentar a los juveniles. En cambio, si se considera el crecimiento de esta especie, entonces es la dieta con $57 \%$ de proteína la que contiene el nivel óptimo. Este estudio es un precedente para realizar nuevas investigaciones en los requerimientos nutricionales de P. adspersus con el fin de mejorar su crecimiento en cautiverio.

Palabras clave: Acuicultura, nutrición, peces, alimentación, indicadores

\section{INTRODUCCIÓN}

El lenguado, Paralichthys adspersus (Steindachner, 1867) es una especie promisoria para su cultivo porque tiene una alta demanda y valor comercial (Alvial \& Manríquez 1999). Esta especie es un recurso sobreexplotado, lo que ha incentivado el desarrollo e implementación de tecnologías para fines comerciales o de repoblamiento tanto en Chile como en Perú (Piaget 2010). Sin embargo, todavía no se han establecido los requerimientos nutricionales ni el nivel óptimo de proteína; aún cuando se ha destacado que es un punto crítico para la crianza de esta especie en cautiverio (Silva 2010). 
El nivel óptimo de proteína dietaria para peces ha sido históricamente determinado utilizando distintas metodologías que consideran el crecimiento (e.g., incremento en peso, tasa de crecimiento) como la variable respuesta en el diseño experimental (Robbins et al. 2006). Indicadores de utilización del alimento (e.g., CA: conversión del alimento, EP: eficiencia proteica), de condición (e.g., K: factor de condición, IHS: índice hepatosomático), o bien indicadores como la tasa de excreción de amonio y de consumo de oxígeno permiten determinar el nivel óptimo de proteína dietaria a incluir (Yigit et al. 2004). En este contexto, Shearer (2000) sugiere integrar al menos dos indicadores para lograr aproximaciones más exactas en la determinación del nivel óptimo de proteína, mientras que Robbins et al. (2006) proponen realizar un análisis de regresión (e.g., línea quebrada, polinomial de segundo orden) para mejorar la precisión en su estimación.

La alimentación de juveniles de Paralichthys adspersus ha sido provista tradicionalmente por dietas formuladas para juveniles de rodaballo o turbot (Psetta maxima Linnaeus, 1758), bajo el supuesto que los peces planos tienen hábitos de vida similares (Alvial \& Manríquez 1999). Antecedentes biológicos referidos al cultivo de Psetta maxima (= Scophthalmus maximus Linnaeus, 1758) han sido descritos y discutidos en la literatura científica, sugiriendo que para un crecimiento óptimo de juveniles es necesario incluir sobre un 50\% de proteína en la dieta (Person-Le Ruyet 2010).

Los juveniles de Paralichthys adspersus alimentados con dietas para Psetta maxima muestran un crecimiento dentro del rango esperado para peces planos en la fase inicial de cultivo (Silva et al. 2001). Sin embargo, en la fase de engorda se hace necesario optimizar el crecimiento de los juveniles, para lo cual es imprescindible determinar el nivel óptimo de proteína en su dieta (Silva 2010). Es en este contexto que el presente estudio fue realizado para establecer el nivel óptimo de proteína en la dieta para obtener el máximo crecimiento de juveniles de $P$. adspersus.

\section{Materiales Y MÉtodos}

Se formularon y elaboraron tres dietas (48, 57 y $60 \%$ de proteína) en base a harina de pescado. Además, se utilizó una dieta comercial para Psetta maxima de BIOMAR S.A. con 54\% de proteína. La formulación, elaboración y conservación de las dietas fue realizada de acuerdo al protocolo estándar del Laboratorio de Nutrición de la Universidad Católica del Norte (UCN) de Coquimbo, basado en AOAC (2000). El análisis proximal y las materias primas utilizadas para la elaboración de las dietas experimentales y de la dieta 54 se presentan en las Tablas 1 y 2.
Tabla 1. Análisis proximal (\% materia seca) de las dietas para juveniles de Paralichthys adspersus (dietas 48, 57 y 60) y de la dieta comercial (54). ELN=Extractos libres de nitrógeno / Proximate analysis (dry matter \%) of Paralichthys adspersus juvenile's diet (diets 48, 57 and 60) and commercial diet (54). ELN=Nitrogen-free extract

\begin{tabular}{lrrrr}
\hline & Dieta 48 & Dieta 54 & Dieta 57 & Dieta 60 \\
\hline Proteína bruta & 47,80 & 53,89 & 57,17 & 59,50 \\
Lípidos totales & 9,85 & 18,16 & 8,82 & 10,34 \\
ELN & 28,44 & 19,57 & 19,22 & 13,65 \\
Cenizas & 13,91 & 8,37 & 14,79 & 16,51 \\
\hline
\end{tabular}

Se utilizaron 156 juveniles marcados (T-Bar anchor tags, Hallprint Fish Tags) provenientes de una misma cohorte (peso promedio 131,82 $\pm 23,25 \mathrm{~g}$, coeficiente de variación = 17,6\%; longitud estándar (Ls) 21,29 $\pm 1,19$ cm, coeficiente de variación $=5,6 \%$ ) producidos en el Laboratorio de Peces de la UCN. Los juveniles fueron aclimatados previamente con la dieta 54. Al inicio del experimento los peces fueron distribuidos, en grupos de 13 individuos, de manera aleatoria en 12 estanques cónicos negros de base plana de $68 \mathrm{~L}$ de capacidad a una densidad inicial de $5,5 \mathrm{~kg} \mathrm{~m}^{-2}$. Cada dieta contó con tres réplicas. Los juveniles fueron alimentados a saciedad dos veces al día (09:00 y 16:00 h), con las dietas experimentales de $6 \mathrm{~mm}$ de diámetro. El experimento se llevó a cabo a temperatura ambiente entre febrero (17,5 \pm $\left.1,1^{\circ} \mathrm{C}\right)$ y julio $2006\left(14,1 \pm 0,5^{\circ} \mathrm{C}\right)$. Durante los 175 días de experimentación, los estanques se mantuvieron con un flujo constante de agua de mar $(34,88 \pm 0,83 \mathrm{psu})$ y con un recambio total por hora. Los peces fueron muestreados para determinar los indicadores de condición y utilización del alimento al inicio y al final del estudio, midiendo la longitud estándar (Ls) con un ictiómetro manual $( \pm 1 \mathrm{~mm}$ de precisión) y el peso total $(\mathrm{Pt}$ ) con una balanza semi-analítica ( \pm 0,1 g de precisión). El crecimiento de los juveniles se evaluó a través de la Tasa de Crecimiento Específico: SGR $=\left((\mathrm{LnLf}-\mathrm{LnLi}) \cdot(\mathrm{Tf}-\mathrm{Ti})^{-1}\right) \cdot 100$, mientras que el porcentaje de supervivencia se determinó mediante el recuento del número inicial y final de juveniles: $\mathrm{S}=\left(\mathrm{N}^{\circ}\right.$ final $\cdot \mathrm{N}^{\circ}$ inicial $\left.{ }^{-1}\right) \cdot 100$ (Silva et al. 2001).

Se calcularon dos indicadores de condición, el índice de Fulton $\left(\mathrm{K}=\right.$ Peso total-Longitud Estandar $\left.{ }^{-3}\right)$ y el índice hepatosomático: $\mathrm{IHS}=$ Peso hígado $\cdot($ Peso total - Peso

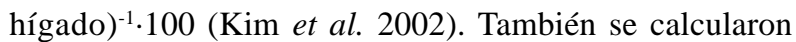
distintos indicadores de utilización del alimento, tales como Conversión del Alimento: CA = alimento seco entregado (g) peso ganado $(\mathrm{g})^{-1}$, Eficiencia del Alimento: $\mathrm{EA}=$ peso ganado $(\mathrm{g}) \cdot$ alimento entregado $(\mathrm{g})^{-1}$, Eficiencia Proteica 
Tabla 2. Ingredientes y contenido energético de las dietas balanceadas de juveniles de Paralichthys adspersus utilizadas en este estudio / Raw material and energy content for Paralichthys adspersus juvenile's diet, used in this study

\begin{tabular}{lrrrr}
\hline Ingredientes $\left({\left.\mathrm{g} 100 \mathrm{~g}^{-1}\right)}^{-}\right.$ & Dieta $48^{1,2,3}$ & Dieta $54^{4}$ & Dieta $57^{1,2,3}$ & Dieta $60^{1,2,3}$ \\
\hline Harina de pescado & 62,95 & 68,00 & 68,42 & 79,36 \\
Aceite de pescado & 6,23 & 5,00 & 5,73 & 4,73 \\
Almidón de maíz & 26,82 & 23,00 & 21,85 & 11,91 \\
Premix vitaminas & 2,00 & 2,00 & 2,00 & 2,00 \\
Premix mineral & 1,00 & 1,00 & 1,00 & 1,00 \\
Vitamina C & 0,50 & 0,50 & 0,50 & 0,50 \\
Colina & 0,50 & 0,50 & 0,50 & 0,50 \\
Contenido energético $\left(\mathrm{Kcal} \mathrm{kg}{ }^{-1}\right)$ & & & & \\
Energía Proteica & 11,29 & 12,72 & 13,49 & 14,02 \\
Energía Lipídica & 3,89 & 7,17 & 3,48 & 4,09 \\
Energía ELN & 4,97 & 3,43 & 3,36 & 2,39 \\
Total Energía Dietaria & 20,15 & 23,32 & 20,34 & 20,49 \\
\hline
\end{tabular}

\footnotetext{
'Harina y aceite de pescado. Pesquera San José S.A.

${ }^{2}$ Premezcla de vitaminas: Suplemento vitamínico para peces (A, D3, K3, E, B1, B2, B6, otros) VETERQUIMICA S.A.

${ }^{3}$ Premezcla de minerales: Suplemento mineral para peces (Mg, Zn, Fe, Cu, I, Co, Se) VETERQUIMICA S.A

${ }^{4}$ Dieta comercial no específica el origen de las materias primas. BIOMAR S.A.
}

$\mathrm{EP}=$ peso ganado $(\mathrm{g}) \cdot$ entrega de proteína seca $(\mathrm{g})^{-1}$, y Retención de Proteína: RP = proteína seca del tejido muscular final (g) · proteína seca del tejido muscular inicial (g)-1 (Yigit et al. 2004). El aporte de energía de las dietas experimentales y la dieta 54 fue calculado de acuerdo al análisis proximal y expresado en Kcal kg-1 (Piaget 2010).

Se determinó la composición proximal de las dietas balanceadas y del músculo de 5 peces antes de iniciar el experimento y de 9 peces por tratamiento al finalizar el experimento. Para determinar el porcentaje de proteína bruta, se estimó el nitrógeno total mediante el método de Kjeldhal, el que se multiplicó por el factor 6,25 (AOAC 2000). Los lípidos totales fueron extraídos con cloroformo-metanol (2:1 v/v) y evaluados gravimétricamente (AOAC 2000). El porcentaje en peso seco se calculó después de secar las muestras a $95^{\circ} \mathrm{C}$ hasta lograr su peso constante. Las cenizas fueron determinadas gravimétricamente tras incinerar las muestras por $15 \mathrm{~h}$ a $500^{\circ} \mathrm{C}$. El extracto libre de nitrógeno (ELN) fue calculado por diferencia de los proximales: 100 - (proteína bruta + lípidos totales + cenizas) (AOAC 2000).

Para determinar el nivel óptimo de proteína en la dieta se utilizaron los análisis de regresión de línea quebrada y polinomial de segundo orden. El análisis de regresión de línea quebrada compara la ganancia en peso de los peces con el nivel de proteína incluido en la dieta. De esta manera se detecta el nivel mínimo de proteína requerido para obtener el máximo crecimiento de los juveniles (Robbins et al. 2006). El análisis de regresión polinomial de segundo orden opera con la ganancia en peso y el nivel proteico dietario, entregando el nivel óptimo y el rango mínimo de proteína requeridos para obtener el máximo crecimiento de los juveniles (Robbins et al. 2006).

Para comparar los indicadores de condición y de utilización del alimento, y el análisis proximal del músculo de los juveniles entre los tratamientos de alimentación se utilizó el análisis de varianza (ANDEVA) de un factor. La normalidad de los datos y la homocedasticidad de las varianzas fueron comprobadas a través de la prueba de Barlett y Lilliefors, y por examinación visual de los residuales para detectar datos ‘fuera de rango’ (Sokal \& Rohlf 1981). La relación entre el peso del hígado y el peso total fue comparada entre tratamientos usando análisis de covarianza (ANCOVA). Cuando el ANDEVA o ANCOVA presentó diferencias significativas se aplicó la prueba a posteriori de Tukey (Sokal \& Rohlf 1981). 


\section{Resultados}

\section{CRECIMIENTO Y SUPERVIVENCIA}

El tamaño de los juveniles de Paralichthys adspersus, expresado en peso total y longitud estándar, mostró un incremento significativo en todos los tratamientos de alimentación al final del experimento (Tabla 3). La SGR es similar entre los peces alimentados con la dieta 57, 60 y 54, y difieren significativamente de la SGR de los peces alimentados con la dieta 48 (ANDEVA $_{(3,72)}=9,44 ; P<$ 0,001 ) (Tabla 3). La supervivencia de los juveniles no fue significativamente distinta entre tratamientos dietarios, variando entre 94 y $100 \%$.

\section{INDICADORES DE CONDICIÓN Y DE UTILIZACIÓN DEL ALIMENTO}

El factor de condición K difiere significativamente entre los peces alimentados con las dietas 48 y 60 (ANDEVA $_{(3,31)}=$ 1,00, $P<0,05)$ (Tabla 3). También, fueron detectadas diferencias significativas en el IHS (ANDEVA $F_{(3,24)}=7,29$, $P<0,001)$. A medida que incrementa la concentración de proteína en la dieta aumenta el tamaño del pez, el cual covaría con el peso del hígado (ANCOVA $\mathrm{F}_{(3,31)}=6,97, P<0,001$ ), repercutiendo en los valores del IHS. En este contexto, los peces alimentados con la dieta 48 presentan un déficit nutricional de proteína, verificado tanto por el valor promedio mínimo del IHS así como por los indicadores de utilización del alimento y el crecimiento de los peces (Tabla 3).

Por otro lado, el superávit proteico contenido en la dieta 60 no favorece el crecimiento de los juveniles en comparación con la dieta 54 o la dieta 57. La dieta 54 entrega una mayor cantidad de energía diaria a los juveniles en comparación con la dieta 57 (Tabla 2), aunque presenta la menor RP (Tabla 3). En cambio, los juveniles alimentados con la dieta 57 presentan una mayor CA y RP, pero con crecimientos similares a los observados en los peces alimentados con la dieta 54 .

Tabla 3. Promedio \pm desviación estándar de crecimiento, supervivencia e indicadores de condición y de utilización del alimento para establecer el nivel óptimo de proteína dietaria en juveniles de Paralichthys adspersus. Los promedios con distintos superíndices presentan diferencias significativas (Prueba de Tukey; $\mathbf{P}<\mathbf{0 , 0 5}$ ) / Average \pm standard deviation of growth, survival and, condition and food utilization indicators to establish optimal dietary protein level for Paralichthys adspersus juveniles. Means with different superscripts are significantly different (Tukey test; $\mathrm{P}<0.05$ )

\begin{tabular}{|c|c|c|c|c|}
\hline & Dieta 48 & Dieta 54 & Dieta 57 & Dieta 60 \\
\hline \multicolumn{5}{|l|}{ Crecimiento y Supervivencia } \\
\hline Peso inicial (g) & $127,37 \pm 21,93$ & $130,23 \pm 23,34$ & $131,88 \pm 24,82$ & $130,23 \pm 20,41$ \\
\hline Peso final $(g)$ & $163,96 \pm 34,95^{\mathrm{a}}$ & $194,02 \pm 45,81^{b}$ & $197,05 \pm 40,01^{b}$ & $209,58 \pm 40,92^{b}$ \\
\hline Longitud inicial $(\mathrm{cm})$ & $21,11 \pm 1,15$ & $21,27 \pm 1,16$ & $21,38 \pm 1,34$ & $21,37 \pm 1,16$ \\
\hline Longitud final $(\mathrm{cm})$ & $23,08 \pm 1,53^{\mathrm{a}}$ & $24,23 \pm 1,38^{b}$ & $23,60 \pm 1,53^{b}$ & $24,65 \pm 1,69^{b}$ \\
\hline Tasa de crecimiento diaria (\% diario) (SGR) & $0,14 \pm 0,02^{\mathrm{a}}$ & $0,23 \pm 0,03^{\mathrm{b}}$ & $0,22 \pm 0,02^{\mathrm{b}}$ & $0,26 \pm 0,02^{\mathrm{b}}$ \\
\hline Número inicial de juveniles (n) & 39 & 39 & 39 & 39 \\
\hline Supervivencia (\%) (S) & $97,40 \pm 4,44$ & 100 & $94,90 \pm 8,88$ & $94,90 \pm 8,87$ \\
\hline \multicolumn{5}{|l|}{ Indicadores de condición } \\
\hline Factor de condición $(\mathrm{K})$ & $1,40 \pm 0,15^{\mathrm{a}}$ & $1,39 \pm 0,09^{\mathrm{ab}}$ & $1,42 \pm 0,12^{\mathrm{ab}}$ & $1,43 \pm 0,09^{\mathrm{b}}$ \\
\hline Índice hepatosomático (\%) (IHS) & $1,16 \pm 0,13^{\mathrm{a}}$ & $1,41 \pm 0,14^{\mathrm{b}}$ & $1,27 \pm 0,16^{\mathrm{ab}}$ & $1,46 \pm 0,15^{\mathrm{b}}$ \\
\hline Peso hígado (g) & $2,22 \pm 0,36^{\mathrm{a}}$ & $3,38 \pm 0,70^{\mathrm{bc}}$ & $3,10 \pm 0,52^{b}$ & $3,56 \pm 0,44^{\mathrm{c}}$ \\
\hline \multicolumn{5}{|l|}{ Indicadores de utilización del alimento } \\
\hline Conversión del alimento (CA) & $1,84 \pm 1,08$ & $2,51 \pm 1,04$ & $2,62 \pm 0,66$ & $2,47 \pm 0,62$ \\
\hline Eficiencia del alimento (EA) & $1,02 \pm 0,13^{\mathrm{a}}$ & $1,56 \pm 0,17^{\mathrm{b}}$ & $1,61 \pm 0,02^{b}$ & $1,84 \pm 0,23^{\mathrm{b}}$ \\
\hline Eficiencia proteica (EP) & $2,12 \pm 0,26^{\mathrm{a}}$ & $2,90 \pm 0,32^{\mathrm{ab}}$ & $2,82 \pm 0,04^{\mathrm{ab}}$ & $3,10 \pm 0,38^{b}$ \\
\hline Retención de proteína (RP) & $1,11 \pm 0,66^{a b}$ & $0,31 \pm 0,26^{\mathrm{a}}$ & $2,65 \pm 1,55^{\mathrm{b}}$ & $2,74 \pm 0,12^{\mathrm{b}}$ \\
\hline
\end{tabular}




\section{COMPOSICIÓN PROXIMAL DEL TEJIDO MUSCULAR}

La concentración de proteína en el tejido muscular de los peces fue significativamente mayor en las dietas $48,57 \mathrm{y}$ 60 en comparación con la dieta 54 (ANDEVA $_{(4,26)}=10,72$, $P<0,001)$ (Tabla 4). En contraste con los peces alimentados con las otras dietas, los peces alimentados con la dieta 54 muestran niveles de proteína en el tejido muscular similar a la muestra inicial (Tabla 4). Además los peces alimentados con la dieta 54 presentaron la máxima concentración de lípidos en el tejido muscular, la que difiere significativamente ANDEVA $\left._{(4,26)}=14,89, P<0,001\right)$ de la concentración de lípidos obtenida en los músculos de los peces alimentados con las dietas experimentales (Tabla 4).

La concentración de ELN en el tejido muscular de los peces, es mínima. Los peces alimentados con la dieta 54 muestran la mayor concentración de ELN, que sólo difiere significativamente de los valores mínimos obtenidos del tejido muscular de los peces al inicio de la experiencia (ANDEVA $_{(4,26)}=4,95, P<0,001$; Tabla 4). El contenido de cenizas en el tejido muscular de los peces difiere significativamente entre las dietas, con un valor promedio máximo detectado al inicio de la experiencia y un mínimo encontrado en los juveniles alimentados con la dieta 54 (ANDEVAF $_{(4,26)}=4,95, P<0,001$; Tabla 4). En general, la menor concentración de proteína en el tejido muscular de los peces alimentados con la dieta 54 es inversamente proporcional a la acumulación de lípidos y ELN.

\section{ANÁLISIS DE REgresión}

De acuerdo al análisis de línea quebrada, el nivel óptimo de proteína está contenido en la dieta 54 (Fig. 1A); mientras que el análisis de regresión polinomial de segundo orden sugiere que este nivel está en la dieta 57 con un valor mínimo de proteína requerida por los juveniles que está sobre lo administrado en la dieta 48 (Fig. 1B).

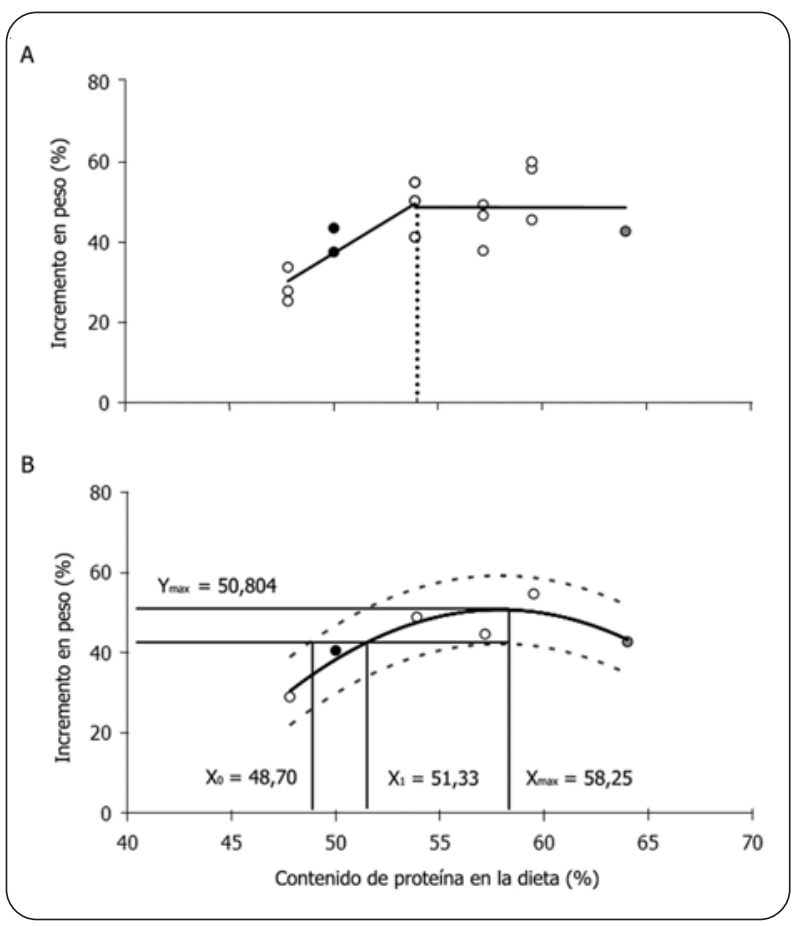

Figura 1. A) Regresión de línea quebrada del nivel óptimo de proteína dietaria para juveniles de Paralichthys adspersus. B) Regresión polinomial de segundo orden del nivel y rango óptimo de proteína dietaria en función del máximo crecimiento de juveniles. Círculos blancos: incremento porcentual en peso de los juveniles en cada réplica por tratamiento de alimentación; círculo negro: información referencial del incremento en peso de los juveniles alimentados con una dieta de $\mathbf{5 0 \%}$ (Silva et al. 2001), y círculo gris: $\mathbf{6 4 \%}$ de proteína (Silva \& Flores 1994) / A) Broken line regression for optimal dietary protein level for Paralichthys adspersus juveniles. B) Second-order polynomial regression for optimal dietary protein level in terms of maximum growth or minimum range of protein requirement for juveniles. White circles: increasing weight percentage of juveniles for each treatment replicate; black circle: information of increasing weight percentage with 50\%protein diet (Silva et al. 2001), and gray circle: $64 \%$ protein (Silva \& Flores 1994 )

Tabla 4. Promedio \pm desviación estándar de los constituyentes proximales (\% materia seca) del tej ido muscular de juveniles de Paralichthys adspersus alimentados con las distintas dietas experimentales ( $\mathbf{n}=\mathbf{9}$ por tratamiento) y al inicio ( $n=5$ ) del experimento. ELN: Extracto libre de nitrógeno. Los promedios con distintos superíndices presentan diferencias significativas (Prueba de Tukey; $\mathbf{P}<\mathbf{0 , 0 5}$ ) / Average \pm standard deviation of proximate constituents (dry matter \%) of muscle tissue from Paralichthys adspersus juveniles fed with different experimental diets ( $n=9$ per treatment) and initial samples $(n=5)$. ELN: Nitrogen-free extract. Means with different superscripts are significantly different (Tukey test; $\mathrm{P}<0.05$ )

\begin{tabular}{lccccc}
\hline & Inicio & Dieta 48 & Dieta 54 & Dieta 57 & Dieta 60 \\
\hline Proteína Bruta & $87,66 \pm 0,40^{\mathrm{b}}$ & $88,77 \pm 0,98^{\mathrm{a}}$ & $87,16 \pm 2,30^{\mathrm{b}}$ & $90,22 \pm 1,53^{\mathrm{a}}$ & $90,40 \pm 1,39^{\mathrm{a}}$ \\
Lípidos totales & $2,78 \pm 0,42^{\mathrm{a}}$ & $4,90 \pm 0,96^{\mathrm{a}}$ & $7,67 \pm 1,80^{\mathrm{b}}$ & $3,21 \pm 1,16^{\mathrm{a}}$ & $3,59 \pm 1,41^{\mathrm{a}}$ \\
ELN & $0,15 \pm 0,08^{\mathrm{ab}}$ & $0,22 \pm 0,06^{\mathrm{ab}}$ & $0,48 \pm 0,29^{\mathrm{b}}$ & $0,79 \pm 0,53^{\mathrm{ab}}$ & $0,51 \pm 0,43^{\mathrm{ab}}$ \\
Cenizas & $8,41 \pm 0,63^{\mathrm{c}}$ & $6,11 \pm 0,37^{\mathrm{b}}$ & $5,54 \pm 0,31^{\mathrm{a}}$ & $5,39 \pm 0,68^{\mathrm{b}}$ & $6,07 \pm 0,26^{\mathrm{ab}}$ \\
\hline
\end{tabular}




\section{Discusión}

Los indicadores de condición, de utilización del alimento y los análisis de regresión indican que las dietas 54 y 57 contienen el nivel óptimo de proteína para alimentar juveniles de Paralichthys adspersus. Silva \& Flores (1994) alimentaron juveniles de la misma edad y peso, con una dieta que contenía $64 \%$ de proteína sin mejorar significativamente el crecimiento en comparación con nuestro estudio (Fig. 1). Mientras que una dieta con $50 \%$ de proteína (Silva et al. 2001), actualmente utilizada en esta etapa del cultivo (Silva 2010), contiene el nivel mínimo de proteína necesario para alimentar juveniles de $P$. adspersus (Fig. 1). No obstante, en Hippoglossus hippoglossus, Psetta maxima, Paralichthys olivaceus y P. californicus, la dieta que contiene el $50 \%$ de proteína fue óptima para alimentar juveniles (Bush 2003, Hamre et al. 2003, Lee et al. 2006, Person-Le Ruyet 2010).

Los juveniles de Paralichthys adspersus alimentados con las dietas 54 y 57 presentaron una tasa de crecimiento semejante. Sin embargo, la dieta 54 es energéticamente alta en comparación con las dietas experimentales (Tabla 2). De acuerdo a Bush (2003) una dieta energéticamente alta contiene sobre $12 \%$ de lípidos totales (e.g., dieta 54; Tabla 1). Dietas de este tipo generan un efecto de ahorro y retención de proteína en los peces debido al reemplazo de esta por lípidos, mejorando las tasas de crecimiento (Kim et al. 2004, González et al. 2005). Por ejemplo, el crecimiento de juveniles de $P$. olivaceus aumenta significativamente cuando son alimentados con dietas que contienen $16 \%$ de lípidos (Lee et al. 2000), aunque dietas por sobre este nivel de lípidos no incrementan el crecimiento (Kim et al. 2004).

Una consecuencia del efecto de ahorro y retención de proteína es el aumento de la concentración de lípidos en el músculo de los peces (Lee et al. 2000), tal como fue detectado en este estudio (Tabla 4). Psetta maxima, así como Paralichthys adspersus, es un pez magro que tiene una baja eficiencia de metabolización de lípidos (Regost et al. 2001, González et al. 2005) y cuando es alimentado con dietas energéticamente altas (16\% lípidos) ocurre una redistribución de los lípidos de reserva desde zonas marginales hacia el tejido muscular e hígado (Regost et al. 2003). En este contexto, Hebb et al. (2003) recomiendan incluir hasta un $10 \%$ de lípidos en la dieta para mejorar el crecimiento de los juveniles sin perjudicar la calidad estructural del tejido muscular. Por lo que la dieta 57 contendría el nivel óptimo de proteína para alimentar juveniles de $P$. adspersus.
Los indicadores de condición muestran una relación directa entre la concentración de proteína y el tamaño de los juveniles de $P$. adspersus. En este contexto, dietas energéticamente bajas con altos niveles proteicos (> 60\%) también producen un efecto de ahorro y retención de proteína (Lie et al. 1988), incrementando el tamaño del hígado debido a su catabolismo (Kim et al. 2002, este estudio). Lo cual explicaría la similitud en el IHS de los peces alimentados con la dieta 54 y la dieta 60.

Los indicadores de utilización del alimento en juveniles de Solea senegalensis muestran que el crecimiento depende del nivel de proteína dietaria (Rema et al. 2008). Sin embargo, la utilización del alimento (e.g., CA, EA) y de la proteína (e.g., EP, RP) en juveniles de $P$. lethostigma, $P$. olivaceus y Psetta maxima disminuyen con el aumento de la concentración de lípidos en la dieta (Lee et al. 2000, Kikuchi \& Takeuchi 2002, Cho et al. 2005, Gao et al. 2005), tal como ocurre con los juveniles alimentados con la dieta 54 (Tabla 3).

Los análisis de regresión fueron una herramienta útil para determinar el rango mínimo y el nivel óptimo de proteína dietaria necesaria para obtener el máximo crecimiento de juveniles de Paralichthys adspersus. El análisis de línea quebrada sugiere que el nivel óptimo de proteína esta incluido en la dieta 54 (Fig. 1A); mientras que el análisis de regresión polinomial indica que el óptimo estaría incluido en la dieta 57 (Fig. 1B). Es en este contexto que Robbins et al. (2006), indican que la selección del nivel óptimo de proteína siempre involucra sopesar los costos biológicos con los productivos, los cuales son informados por los indicadores de condición y de utilización del alimento (Shearer 2000).

El nivel óptimo de proteína incluido en la dieta varía en función de la ontogenia del pez, la época y lugar de crianza (Lee et al. 2000, Hartlen et al. 2005). Adicionalmente, la calidad de la proteína incluida en la dieta también puede modificar los resultados (Regost et al. 2001, Yigit et al. 2004, este estudio); así como los factores extrínsecos asociados a la tecnología de cultivo (e.g., variables ambientales) (Piaget 2010). Por estas razones, decidir cuál es la concentración de proteína óptima que debe incluirse en la dieta para obtener el mejor crecimiento de juveniles de Paralichthys adspersus, dependerá de los intereses que llevan a realizar la crianza.

En conclusión, las dietas 48 y 60 afectan el crecimiento de los juveniles por déficit o superávit de proteína; mientras que las dietas 54 y 57 son las que presentan el nivel óptimo 
para un adecuado crecimiento. Sin embargo, para discriminar entre estas dietas, se requieren nuevos estudios que determinen la proporción óptima de proteína y lípidos a incluir. Por otra parte, la decisión del «óptimo de proteína» para el adecuado crecimiento de los juveniles está relacionada con costos biológicos o productivos (Piaget 2010). En este contexto, como la acuicultura es una actividad productiva que busca máximos niveles de producción a costos mínimos (Silva 2010), la dieta 54 es óptima para alimentar juveniles de Paralichthys adspersus.

Finalmente, este estudio determinó el óptimo de proteína para alimentar juveniles en etapa de engorda (peces de 120$130 \mathrm{~g}$ ), por lo que nuevos análisis deben ser enfocados a establecer los óptimos proteicos en la etapa larvaria, de preengorda y reproductores de Paralichthys adspersus, considerando que el uso de distintos indicadores mejora la exactitud para discriminar la dieta óptima. Estudios complementarios deben ser enfocados a prescribir la composición de aminoácidos, principalmente del grupo de los esenciales, puesto que son determinantes para el crecimiento de peces en cultivo (Regost et al. 2001); y a la calidad y cantidad de lípidos dietarios debido a las consecuencias fisiológicas que conlleva su inclusión (Regost et al. 2003, Gao et al. 2005), lo que a futuro permitirá el desarrollo de dietas con fuentes proteicas de menor costo pero adecuadas para la alimentación de $P$. adspersus en cautiverio.

\section{Agradecimientos}

Los autores agradecen al personal del Laboratorio de Peces, de Nutrición, de Oceanografía y de la Unidad de Producción de la Facultad de Ciencias del Mar de la UCN, especialmente a M. Oliva, M. López y S. Fuentes. También agradecemos los comentarios y correcciones de C. López y dos evaluadores anónimos que mejoraron sustancialmente el contenido de este manuscrito. Este estudio es parte de la tesis de Magíster en Ciencias del Mar del primer autor (NP). AV agradece la beca de doctorado del GORE Coquimbo, Facultad de Ciencias del Mar, UCN y CEAZA.

\section{LITERATURA CITADA}

Alvial A \& J Manríquez. 1999. Diversification of flatfish culture in Chile. Aquaculture 176: 65-73.

AOAC. 2000. Official methods of analysis of the association of official analytical chemist, 870 pp. Association of Analytical Chemist, Arlington.

Bush D. 2003. The effect of dietary lipid content on protein utilization in California Halibut, Paralichthys californicus.
Thesis of Master of Science, University of California, Davis, 97 pp.

Cho SH, SM Lee, SM Lee \& JH Lee. 2005. Effect of dietary protein and lipid levels on growth and body composition of juvenile turbot (Scophthalmus maximus L) reared under optimum salinity and temperature conditions. Aquaculture Nutrition 11: 235-240.

Daniels HV \& ML Gallagher. 2002. North American flounders. En: Webster C \& C Lim (eds). Nutrient requirements \& feeding of finfish for aquaculture, pp. 121130. CAB International, Oxford.

Gao Y, J Lv, Q Lin \& L Li. 2005. Effect of protein levels on growth, feed utilization, nitrogen and energy budget in juvenile southern flounder, Paralichthys lethosthigma. Aquaculture Nutrition 11: 427-433.

González S, SR Craig, E McLean, MH Schwarz \& GJ Flick. 2005. Dietary protein requirement of southern flounder, Paralichthys lethostigma. Journal of Applied Aquaculture 17: 37-50.

Hamre K, A Øfsti, T Næss, R Nortvedt \& JC Holm. 2003. Macronutrient composition in formulated diets for Atlantic halibut (Hippoglossus hippoglossus L.) juveniles, a multivariate approach. Aquaculture 227: 233-244.

Hartlen B, B Grisdale-Helland \& S Helland. 2005. Growth, feed utilization and body composition in two size groups of Atlantic halibut (Hippoglossus hippoglossus) fed diets differing in protein and carbohydrate content. Aquaculture 249: 401-408.

Hebb CJ, D Castell, D Aderson \& J Batt. 2003. Growth and feed conversion of juvenile winter flounder (Pleuronectes americanus) in relation to different protein to lipid levels in isocaloric diets. Aquaculture 22: 439449.

Kikuchi K \& T Takeuchi. 2002. Japanese flounder, Paralichthys olivaceus. En: Webster C \& C Lim (eds). Nutrient requirements and feeding of finfish for aquaculture, pp. 113-120. CAB International, Oxford.

Kim K, X Wang \& SC Bai. 2002. Optimum dietary protein level for maximum growth of juvenile olive flounder Paralichthys olivaceus (Temminck et Schlegel). Aquaculture Research 33: 673-679.

Kim K, X Wang, S Choi, G Park, SC Bai. 2004. Evaluation of optimum dietary protein-to-energy ratio in juvenile olive flounder Paralichthys olivaceus (Temminck et Schlegel). Aquaculture Research 35: 250-255.

Lee SM, SH Cho \& KD Kim. 2000. Effects of dietary protein and energy levels on growth and body composition of juvenile flounder Paralichthys olivaceus. Journal of World Aquaculture Society 31: 306-315.

Lee S, JH Lee, K Kim \& SH Cho. 2006. Optimum dietary protein for growth of juvenile starry flounder, Platichthys stellatus. Journal of World Aquaculture Society 37: 200203. 
Lie O, E Lied \& G Lambertsen. 1988. Feed optimization in Atlantic cod (Gadus morhua): fat versus protein content in the feed. Aquaculture 69: 333-341.

Person-Le Ruyet J. 2010. Turbot culture (Scophthalmus maximus). En: Daniels HV \& WO Watanabe (eds). Practical flatfish culture and stock enhancement, pp. 392412. Wiley-Blackwell, Ames.

Piaget N. 2010. Determinación del nivel óptimo de proteína dietaria en juveniles del lenguado, Paralichthys adspersus (Pisces, Pleuronectiformes: Paralichthyidae). Tesis de Magíster en Ciencias, Facultad de Ciencias del Mar, Universidad Católica del Norte, Coquimbo, 101 pp.

Regost C, J Arzel, M Cardinal, J Robin, M Laroche \& SJ Kaushik. 2001. Dietary lipid level, hepatic lipogenesis and flesh quality in turbot (Psetta maxima). Aquaculture 193: 291-309.

Regost C, J Arzel, JH Robin, G Rosenlund \& SJ Kaushik. 2003. Total replacement of fish oil by soybean or linseed oil with a return to fish oil in turbot (Psetta maxima): growth performance, flesh fatty acid profile and lipid metabolism. Aquaculture 217: 465-482.

Rema P, LE Conceicao, F Evers, M Castro-Cunha, MT Dinis \& J Días. 2008. Optimal protein dietary levels in juvenile Senegalese sole (Solea senegalensis). Aquaculture Nutrition 14: 263-269.
Robbins KR, AM Saxton \& LL Southern. 2006. Estimation of nutrient requirements using broken-line regression analysis. Journal of Animal Science 84: 155-165.

Shearer KD. 2000. Experimental design, statistical analysis and modeling of dietary nutrient requirement studies for fish: a critical review. Aquaculture Nutrition 6: 91-102.

Silva A. 2010. Culture of Chilean flounder. En: Daniels HV \& WO Watanabe (eds). Practical flatfish culture and stock enhancement, pp. 30-45. Wiley-Blackwell, Ames.

Silva A \& H Flores. 1994. Observations on the growth of the Chilean flounder (Paralichthys adspersus, Steindachner, 1867) in captivity. European Aquaculture Society, Special Publication 22: 323-331.

Silva A, M Oliva \& F Castelló. 2001. Evaluación del crecimiento de juveniles de lenguado chileno (Paralichthys adspersus, Steindachner, 1867) cultivado en estanques. Biología Pesquera 29: 21-30.

Sokal RR \& FJ Rohlf. 1981. Biometry: Principles and practice of statistical in biological research, 776 pp. Freeman WH \& Company, San Francisco.

Yigit M, S Cosió, S Teshima \& M Ishikawa. 2004. Dietary protein and energy requirements of juvenile Japanese flounder, Paralichthys olivaceus. Journal of Applied Sciences 4: 486492.

Recibido el 17 de agosto de 2010 y aceptado el 30 de noviembre de 2010 